\title{
UNA NOTA SOBRE ADAM SMITH, ULLOA Y LA ECONOMIA DE BUENOS AIRES
}

\author{
CARLOS NEWLAND y DANIEL WAISSBEIN * \\ Universidad de Oxford
}

En su Riqueza de las Naciones, Adam Smith ilustra frecuentemente los principios generales que, según él, explican el acontecer económico con los ejemplos más variados. De ahí que su monumental obra puede considerarse un verdadero tratado de historia económica, en el que se analizan aspectos de las economías de la China, el Perú, Japón, Francia, España y Rusia, entre otras. Tampoco Buenos Aires y la economía del Plata escaparon a su examen. Pero antes de exponer sus ideas sobre la economía pampeana, daremos unas breves indicaciones sobre la fuente que permitió que Smith conociese algunos datos importantes sobre esa remota región de la Corona española.

Se trata de la obra de Antonio de Ulloa Relación bistórica del viage a la América Meridional becho por don Jorge Juan [...] y don Antonio de Ulloa, publicada en Madrid en $1748^{1}$. El enorme interés que despertó en Europa lo atestigua el hecho de que la primera traducción francesa haya aparecido apenas cuatro años más tarde, y que la versión inglesa, a la que siguieron numerosas reimpresiones, se publicase por primera vez en $1758^{2}$. La quinta

- Los autores agradecen a Guido di Tella y a Leandro Prados de la Escosura sus comentarios sobre esta nota.

I Su título completo es: Relación histórica del viage a la America Meridional hecho por orden de Su Magestad para medir algunos grados de meridiano terrestre, $y$ venir por ellos en conocimiento de la verdadera Fioura y Magnitud de la Tierra, con otras varias observaciones Astronómicas, y Phisicas: Por don Jorge Juan [sigue una larga lista de sus títulos] y don Antonio de Ulloa [ídem], impressa de orden del Rey Nuestro Señor, en Madrid, por Antonio Marín, Año de M.D.CC.XLVIII.

$2 \mathrm{Y}$ no en 1807 como parecen indicar Campbell, Skinner y Tood en la edición del bicentenario de La Riqueza de las Naciones: Adam Smith, An Inquiry into the Nature and Causes of the Wealth of Nations (Oxford, 1976). La traducción inglesa lleva por título: $A$ Voyage to South-America: Describing at large the Spanish Cities, Towns, Provinces, etc. on that Extensive Continent Interspersed throughout with reflections on the Genius, Customs, Manners and Trade of the Inhabitants; together with the Natural History of the Country and an Account of their Gold and Silver Mines undertaken by Command of his Majesty the King of Spain by Don George Juan, and Don Antonio de Ulloa Both Captains of the Spanish Navy, Members of the Royal Societies of London and Berlin, and Corresponding members of the Royal Academy at Paris. Translated from the original Spanish. Illus- 
edición londinense, de 1806, está dedicada al comodoro Sir Home Popham, que, cuando comandaba las fuerzas británicas en El Cabo, cruzó el Atlántico y ocupó brevemente Montevideo y Buenos Aires. El impresor justificaba su dedicatoria explicando que el libro contenía «entre otros asuntos interesantes una breve descripción de Buenos Ayres, ese territorio que ahora es y muy probablemente seguirá siendo, gracias a la Previsión, Habilidad y Valentía personal de Usted, una de las joyas más espléndidas de la Corona Unida» ${ }^{3}$.

Fue precisamente esa «breve descripción de Buenos Ayres» uno de los pasajes que habían llamado la atención de Smith más de cuarenta años antes. Sin embargo, ni Juan ni Ulloa visitaron la región del Plata en el largo viaje que hicieron por las regiones meridionales del continente. Habian zarpado de Cádiz en 1735 y, en casi diez años de misión científica en compañía de sabios franceses, visitaron regiones que hoy forman parte de Colombia, Venezuela, Panamá, Ecuador, Perú, Bolivia y Chile. El viaje acabó con el regreso de Ulloa a Madrid a mediados de 1746 , tras su captura en alta mar por un buque británico y su prisión en Inglaterra. Durante su período de residencia obligada en Londres fue elegido miembro de la Royal Society, en honor a sus méritos científicos.

A diferencia de Alonso Carrió de la Vandera, "Concolorcorvo", autor del Lazarillo de Ciegos Caminantes, itinerario de un viaje en carreta de Buenos Aires a Lima, y que también había llegado a América por primera vez hacia 1735, la descripción que Ulloa hace de Buenos Aires no es, como ya lo indicamos, de primera mano ${ }^{4}$. Pero fueron tan exhaustivas las precauciones tomadas por Ulloa para asegurarse del carácter fidedigno de las noticias que transmite sobre las regiones no visitadas que Adam Smith debió pensar, con entera razón, que merecían toda confianza ${ }^{5}$.

Ulloa, después de describir Nuestra Señora de Buenos-Ayres y de referirse a su fundación, aspecto y clima, indica:

trated with Copper Plates. In two volumes. London. Printed for L. Davis and C. Reymers. Against Gray's-Inn Gate, Holborn, MDCCLVIII.

${ }^{3}$ Se trata de la quinta edición aparecida en 1807. La dedicatoria está fechada el 26 de septiembre de 1806.

" $\mathrm{Si}$ bien "Concolorcorvo" no inició el viaje que describe en su libro hasta 1771.

"Aun cuando Ulloa, a quien cupo escribir "todo to perteneciente a Historia y Sucessos del Viage", nos dice en el prólogo que "no se habla de pasage donde no hayamos estado y residido algún tiempo", él mismo concede que "si se hace de otros, por donde no transitamos, como sucede con [...] los corregimientos del Virreinato de Lima, es siguiendo para ello el más aprobado dictámen [...] en la extensión y pueblos [...] de los Curas, y governadores de ellos, y en Historia Natural, de unos y otros con quienes mantuvimos correspondencia: por cuyo medio, y el celo con que estos deseaban concurrir al cumplimiento de los preceptos del Real agrado, daban puntual satisfacción a nuestras preguntas, aclarando las dudas en que nos dexaban algunas respuestas, con otras". Ullos, op. cit. (1748) i, follo 3. 
"Circundan a la Ciudad unas Campañas muy dilatadas y amenas, donde se estiende la vista sin embarazo para lograr el recreo de la verde Yerva, que las viste con igualdad: su fertilidad hace, sean las Carnes tan abundantes, que su valor por lo inferior no puede compararse con el que tienen en la Ciudad mas abastecida, y commoda de Europa, o de las Indias; siendo el Cuero de las Reses el que ordinariamente se compra: a esto se agrega su buen gusto, porque estando siempre gordas, nunca dexa de ser delicado. Las Campañas, que se estienden de Buenos-Ayres para el Occidente, y Sur, y acia el Norte, estaban ahora 20. años tan pobladas de Ganados Bacuno y Cavallar silvestre, que su mayor costo consistía en el trabajo de irlo a coger, y despues se vendía un Cavallo por un peso de aquella Moneda; y una Baca escogida en Tropa de 200. o 300. por quatro reales. Aunque en los tiempos presentes no faltan, no es con aquella abundancia, y estan algo mas retirados por las crecidas matanzas, que tanto los Españoles, como los Portugueses han hecho para aprovecharse de la Corambre, principal renglón de aquel Comercio.

A proporción de las Carnes es abundante de Caza, y no menos de Pescado, el qual se logra en aquel Rio de varias especies, particularizandose la de los Pexe-Reyes, que los hay de media vara, y aun algo mas de largo. En Frutas es tambien muy abastecida, lograndose de las Europeas, y Criollas; y por todo lo que se ha referido, es un País de los mas regalados, que se pueden imaginar para las comodidades de la vida, haciendo excesso sobre todo la sanidad del Ayre, que en el corre» ${ }^{6}$.

Esta descripción fue utilizada por Smith para ilustrar su teoría de las etapas, por la cual debían transitar todas las sociedades. El primer período fue denominado por Smith "de cazadores», en el cual la población se sustenta mediante la recolección de los frutos espontáneos de la tierra, y sus actividades principales son la pesca y la caza, que en el caso concreto de Buenos Aires incluía la caza del ganado salvaje. La propiedad privada no aparece hasta el segundo período, el "pastoril», en que el ganado pasa a tener dueño y es desplazado siempre en busca de mejores pasturas naturales. Con el advenimiento de la «agricultura» se inicia la tercera etapa: la tierra es apropiada por individuos que exigen una renta por su utilización. El ciclo se cierra con la fase «comercial»: la moneda reemplaza al trueque e impera la división de]

- Ibid., iii, 243-244. 
trabajo, que permite el incremento de la producción y el consumo de una mayor variedad de bienes ?.

Para Smith, Buenos Aires era, sin lugar a dudas, una sociedad en la primera fase, que pasaría lentamente a la tercera fase; es decir, de una economía de caza a una economía agrícola. En el período inicial, las tierras no estaban pobladas y el ganado vacuno se reproducía libremente. El costo de una res se limitaba al trabajo necesario para atraparla, de lo cual resultaba una abundancia de carne:

«Pero en países casi desolados o muy parcamente habitados; como la ganadería, las aves domésticas, la caza de todo tipo, etc., son producto espontáneo de la naturaleza, ésta los produce a menudo en cantidades mucho mayores a las que el consumo de los habitantes requiere. En tal estado de cosas la oferta habitualmente excede la demanda" ${ }^{8}$.

Smith comenta el bajo costo de la carne en Buenos Aires; según Ulloa, se pagaban cuatro reales por una buena res, que al cambio de la época representaban 21 peniques y medio. Smith se refiere, claro está, a la época en que Ulloa efectuó su viaje (1735-1744), y si el precio que menciona es correcto, una res en Londres costaba entonces aproximadamente 70 veces más ${ }^{9}$.

Según el escocés, en la primera etapa (la de caza), una libra de carne tiene un precio menor que una libra de pan. La explicación consiste en que producir grano requiere relativamente mucho más trabajo que cazar y carnear vacunos. Precisamente ésta es la razón, acotamos, por la cual los agricultores en el Plata tuvieron inicialmente dificultades para encontrar mano de obra barata, y fue necesario, por ejemplo, que en 1792 el Cabildo obligase a indios, mulatos y negros a efectuar tareas agrícolas, forzándolos a aceptar un salario menor al que ofrecía la ganadería ${ }^{10}$. Tal situación reflejaba, sin duda, la distinta productividad de ambas actividades. La tierra, al ser muy abundante, no requería renta $\mathrm{y}$, por lo tanto, únicamente el trabajo intervenía en la composición del precio:

7 A. SkINNER, "Adarn Smith: An Economic Interpretation of History", en A. Skinner y T. Wilson (eds.), Essays on Adam Smith (Oxford, 1975), pp. 154-178.

- Smrri, op. cit., 1,206 (la traducción de ésta y de las demás citas fue hecha por nosotros).

- El precio de una res de 700 libras, en Londres en 1733, era de aproximadamente 116 chelines (un chelín=doce peniques). Véase LORD BEvERIDGE, Prices and Wages in England (London, 1965), i, 732.

10 R. LEvene, "Riqueza, industrias y comercio durante el Virreynato", en R. Levene (ed.), Historia de la nación argentina (Buenos Aires, 1940), iv, 274. 
«Pero los valores de estos dos tipos de alimentos, el pan y la carne, son muy diferentes en los distintos periodos de la agricultura. En sus rudos comienzos, las regiones agrestes que entonces ocupan la mayor parte del país, están todas abandonadas al ganado. Hay más carne que pan, y el pan, por lo tanto, es el alimento por el cual existe mayor competencia y que en consecuencia obtiene el precio más elevado. En Buenos Ayres, cuenta Ulloa, cuatro reales, veintiún peniques y medio, era, cuarenta o cincuenta años atrás, el precio ordinario de un buey, escogido en una tropilla de doscientos o trescientos. No nos dice nada sobre el precio del pan, probablemente porque no halló nada digno de nota al respecto. Un buey allí, dice, cuesta poco más que el trabajo de capturarlo. Pero el grano no puede cultivarse en ninguna parte sin un trabajo considerable, y en un país que yace al margen del Río de la Plata, en esa época el camino directo de Europa a las minas de Plata del Potosí, el precio monetario del trabajo no podía ser muy bajo" ".

Con el transcurso del tiempo, y al aumentar la población, el trabajo sería más abundante y mayor la demanda de alimentos. La tierra se comenzaría a cultivar cada vez más, extendiéndose continuamente la zona sembrada. El factor tierra pronto dejaría de ser gratuito - sin usos alternativos-y comenzaría a exigir una renta según su fertilidad o su proximidad a las ciudades y a los caminos. Estas predicciones de Smith se cumplieron rigurosamente: en 1755 fue necesario emitir un bando, en Buenos Aires, que limitaba la extensión de las chacras en defensa de los intereses de los ganaderos ${ }^{12}$. En 1790, la decadencia de la ganadería parecía irremediable, y un estudio del Cabildo indicaba como una de sus causas el «abuso de sembrarse trigo, maíz y otras especies en las mismas estancias, de donde resultaban ahuyentados los ganados» ${ }^{13}$.

La renta, según Smith, reflejaba la escasez de la tierra, y la producción de ganado tenía que competir ahora con la agricultura por la utilización de ese factor:

«Debido a la extensión de los cultivos, las regiones agrestes se vuelven insuficientes para abastecer la demanda de carne. Una gran parte de las tierras cultivadas debe emplearse en criar y engordar el ganado, cuyo precio, por lo tanto, debe ser suficiente

$"$ SmrTh, op. cit., i, 164.

12 LeVene, op. cit., p. 164.

13 Citado en Levene, op. cit., p. 266. 
para pagar, no sólo el trabajo necesario para criarlo sino también la renta que el propietario y el beneficio que el agricultor podrían haber recibido si hubiesen dedicado esa tierra a la labranza» ${ }^{14}$.

Tal estado de cosas llevaría progresivamente a un cambio en los precios relativos del pan y de la carne. La liḅra de carne costaría, en la nueva situación, más que la libra de pan:

«Es de otra manera cuando los cultivos se extienden sobre la mayor parte del país. Hay entonces más pan que carne. La competencia cambia su dirección, y el precio de la carne pasa a ser mayor que el del pan» ${ }^{15}$.

Otro hecho que atrajo la atención de Smith fue que se sacrificase el ganado para utilizar solamente el cuero y el sebo:

«En algunas provincias de España, me han asegurado, se sacrifica muchas veces una oveja para quedarse apenas con el vellón y el sebo. El resto es frecuentemente abandonado y se pudre a la interperie, o es devorado por las bestias y las aves de rapiña. Si esto ocurre en ocasiones aún en España, ocurre casi constantemente en Chile, Buenos Ayres y en muchas otras partes de la América Española, donde casi siempre se mata el ganado vacuno por su cuero y sebo" ${ }^{16}$.

Smith inserta esta observación en su teoría de las etapas, y la explica así: en los países en que existe ganado salvaje en abundancia, el cuero y el sebo tienen un valor muy superior al resto del animal. Ocurre así porque ésas son las únicas partes que pueden exportarse fácilmente. Con el paso del tiempo, al aumentar la población, también aumenta la demanda de carne, elevando su precio. No pasa lo mismo con el cuero y el sebo, ya que su precio es determinado por el mercado internacional y no se ve afectado por un aumento relativamente pequeño de la demanda interna de un país:

"Aunque con el progreso de las artes y de la población, el precio de la bestia entera aumenta inevitablemente, el precio por el resto del animal ha de ser afectado mucho más por este aumento, con toda probabilidad, que el de la lana y del cuero. El mer-

14 SmIrн, op. cit., i, 165.

is Ibid., p. 164.

16 Ibid., p. 247. 
cado por el resto del animal, que en el rudo comienzo de la sociedad está siempre confinado al país que lo produce, aumenta necesariamente con el crecimiento de la población del país que la produce, $y$ en proporción a las mejoras y población de ese país; pero como el mercado por la lana y el cuero, aún en los países bárbaros, se extiende a menudo a todo el mundo comercial, muy pocas veces puede aumentar en la misma proporción. El estado de todo el mundo comercial pocas veces puede verse muy afectado por el crecimiento de un país determinado; y el mercado para esos productos puede permanecer igual o casi igual, después del crecimiento, a lo que era antes de que éste ocurriera» ${ }^{17}$.

Esta cita corresponde al último de los párrafos que dedica Adam Smith a la economía del Río de la Plata, a la que cupo así tener al pensador escocés como el primer economista académico que investigó algunos de sus aspectos más característicos.

\footnotetext{
17 Idem.
} 